\title{
Editorial: Cutaneous T-Cell Lymphomas
}

\author{
Catherine G. Chung ${ }^{1,2^{*}}$ and Basem M. William ${ }^{3}$ \\ 1 Departments of Pathology, The Ohio State University Wexner Medical Center and The James and Solove Cancer Hospital, \\ Columbus, $\mathrm{OH}$, United States, ${ }^{2}$ Medicine, Division of Dermatology, The Ohio State University Wexner Medical Center and \\ The James and Solove Cancer Hospital, Columbus, $\mathrm{OH}$, United States, ${ }^{3}$ Division of Hematology, The Ohio State University \\ Wexner Medical Center and The James and Solove Cancer Hospital, Columbus, OH, United States
}

Keywords: cutaneous T cell lymphoma, brentuximab, PD-L1, mycosis fungoides, CAR T cell

\section{Editorial on the Research Topic}

Cutaneous T-Cell Lymphomas

Cutaneous T-cell lymphomas (CTCL), mycosis fungodies (MF) and its leukemic counterpart Sezary syndrome (SS), remain incurable malignancies with significant impact on patients' quality of life (1). These are however exciting times for clinicians caring for patients with CTCL with approval of two highly effective agents in the past few years. Brentuximab vedotin (BV), a chimeric anti-CD30 monoclonal antibody conjugated to monmethyl auristatin E (MMAE) toxin, was approved by the US Food and Drug Administration (FDA) in 2017 based on the results of the phase III study (ALCANZA) that showed a superior overall response, lasting 4 months of $56.3 \%$ as compared to $12.5 \%$ for physician's choice of methotrexate or bexarotene in patients with relapsed CTCL (2). Mogamulizumab, a humanized IgG1 anti-CCR4 monoclonal antibody, was also approved by the FDA in 2018, based on the phase III study (MAVORIC) that showed superior progression-free survival of mogamulizumab of 7.7 compared to 3.1 months with vorinostat in patients with relapsed CTCL (3).

In this Research Topic of Frontiers in Oncology focused on CTCL, Khan and Sawas review ongoing studies of antibody-directed therapies for MF/SS and the rationale for their use; among them are PD-1 and PD-L1 inhibitors nivolumab and pembrolizumab, both of which have had increasing applications in the treatment of various solid and hematologic malignancies. PD-L1 is expressed in a subset of patients with MF/SS, and pembrolizumab has shown a promising ORR of $38 \%$ in patients, in CITN-10 trial, with relapsed CTCL with a subset of patients experiencing very durable responses (4). Despite the emergence of multiple new targeted agents for the treatment of CTCL, skin-directed therapy remains the mainstay of treatment for early disease, and is reviewed in this issue by Tarabadkar and Shinohara, including current evidence and updated management recommendations of United States Cutaneous Lymphoma Consortium (USCLC).

The pathogenesis of CTCL remains incompletely understood and largely unknown. In this issue, several authors lend insights to our current understanding of lymphomagenesis in MF/SS. Ghazawi et al. reviews recent epidemiological data evaluating risk factors in the development of CTCL, including sex, age, race and various environmental, infectious, and iatrogenic exposures that shed light on possible mechanisms of malignancy in MF/SS. Gantchev et al. reports on the aberrant activation of meiosis genes in cells undergoing mitosis (a process termed "meiomitosis") in the setting of CTCL, and demonstrate that there is differential gene expression of meiosis-specific cancer testis (meiCT) genes in a cohort of SS patients compared to healthy controls; these findings suggest that malignant cells in SS undergo meiomitosis, which may allow for the development of 
novel diagnostic tests that accurately distinguish CTCL from benign inflammatory conditions, as well as targeted therapies. Ferranti et al. discuss immunomodulation in CTCLs, and note that in a recent investigation of HIV-infected and non-HIVinfected patients with MF/SS, individuals with HIV demonstrated significantly higher survival and decreased risk of overall mortality compared to those without HIV. The authors also discuss exploiting retrovirus infection mechanisms for gene therapy in the treatment of CTCL, and review current studies to this end. In addition to identifying specific genetic aberrations in MF/SS involving TP53, the NFkB pathway, and the JAK3/ STAT3 signal transduction pathways that may provide specific targets for future therapies, these technologies have led to the identification of multiple distinct subpopulations with different drug sensitivities within a single patient (5), suggesting that the ideal treatment for CTCL may involve combination therapies informed by a patient's unique malignant $\mathrm{T}$-cell population. Phyo et al. summarizes new findings in the understanding of the biology of CTCL based on newer technologies that allow more precise molecular investigations of malignant $\mathrm{T}$-cells including whole genome and whole exome sequencing and single cell RNA sequencing The evolving role of chimeric

\section{REFERENCES}

1. William BM, Vose JM. T-Cell Lymphomas. Lymphoma. Humana Press, Totowa, NJ: Springer (2013) p. 211-29.

2. Prince HM, Kim YH, Horwitz SM, Dummer R, Scarisbrick J, Quaglino P, et al. Brentuximab vedotin or physician's choice in CD30-positive cutaneous T-cell lymphoma (ALCANZA): an international, open-label, randomised, phase 3, multicentre trial. Lancet (Lond Engl) (2017) 390(10094):555-66. doi: 10.1016/ S0140-6736(17)31266-7

3. Kim YH, Bagot M, Pinter-Brown L, Rook AH, Porcu P, Horwitz SM, et al. Mogamulizumab versus vorinostat in previously treated cutaneous T-cell lymphoma (MAVORIC): an international, open-label, randomised, controlled phase 3 trial. Lancet Oncol (2018) 19(9):1192-204. doi: 10.1016/ S1470-2045(18)30379-6

4. Khodadoust MS, Rook AH, Porcu P, Foss F, Moskowitz AJ, Shustov A, et al. Pembrolizumab in Relapsed and Refractory Mycosis Fungoides and Sézary antigen receptor (CAR) T-cell therapy is summarized by Scarfo et al. highlighting the unique challenges in applying CAR-T cell therapy in the setting of T-cell malignancies including the consequences of T-cell aplasia and the killing of CARexpressing cells by each other; a phenomenon described as "fratricide."

This Research Topic of Frontiers in Oncology provides an overview of key issues relating to the pathobiology, current, and future, management of CTCL and should help identify areas of common interest between dermatologist, hematologists, and cutaneous biologists thus encouraging collaboration in both basic science research and translation into practice through national and international clinical trials. We hope you find this issue interesting and informative.

\section{AUTHOR CONTRIBUTIONS}

All authors listed have made a substantial, direct, and intellectual contribution to the work and approved it for publication.

Syndrome: A Multicenter Phase II Study. J Clin Oncol (2020) 38(1):20-8. doi: 10.1200/JCO.19.01056

5. Buus TB, Willerslev-Olsen A, Fredholm S, Blümel E, Nastasi C, Gluud M, et al. Single-cell heterogeneity in Sézary syndrome. Blood Adv (2018) 2(16):2115-26. doi: 10.1182/bloodadvances.2018022608

Conflict of Interest: The authors declare that the research was conducted in the absence of any commercial or financial relationships that could be construed as a potential conflict of interest.

Copyright (c) 2021 Chung and William. This is an open-access article distributed under the terms of the Creative Commons Attribution License (CC BY). The use, distribution or reproduction in other forums is permitted, provided the original author(s) and the copyright owner(s) are credited and that the original publication in this journal is cited, in accordance with accepted academic practice. No use, distribution or reproduction is permitted which does not comply with these terms. 